\title{
Performance Comparison of Various controllers on Semi-Active Vehicle Sus- pension System
}

\author{
Sarvesh Walavalkar ${ }^{1, *}$, Viraj Tandel ${ }^{1, * *}$, Rahul Sunil Thakur $^{1, * * *}$, V.V Pramod Kumar $^{1, * * * *}$, and Supriya Bhuran ${ }^{1, \dagger}$ \\ ${ }^{1}$ Ramrao Adik Institute of Technology, Nerul, Navi Mumbai 400706, Maharashtra, India
}

\begin{abstract}
The value of a self-tuning adaptive semi-active control scheme for automotive suspension systems is discussed in this paper. The current vehicle suspension system uses fixed-coefficient springs and dampers. The ability of vehicle suspension systems to provide good road handling and improve passenger comfort is usually valued. Passive suspension allows you to choose between these two options. Semi-Active suspension(SAS), on the other hand, can provide both road handling and comfort by manipulating the suspension force actuators directly. The semi-active suspension system for a quarter car model is compared to passive and various controllers such as Proportional-Integral, Proportional-Integral-Derivative, Internal model control (IMC)-PID, IMC-PID with filter, FUZZY, and Adaptive-network-based fuzzy inference system(ANFIS) in this analysis. This research could be relevant in the future for designing better car suspension adjustments to eliminate vertical jerks and rolling motion experienced by the vehicle body on bumps and humps.
\end{abstract}

\section{Introduction}

\subsection{Motivation and background}

Suspension systems are vital for vehicles' health and their life expectancies, and also for rider's health, safety, and comfort. The chore of the vehicle suspension system is to ensure ride comfort by alienating unwanted disturbances in various conditions The current passive suspension systems limit the motion of the Vehicle's body and wheel by limiting their relative velocities to a rate that fails to provide the required ride comfort. So, developing and designing an advanced controller for semi-active suspension systems will reinforce the existing semi-active suspension systems and provide better resistance against the vertical acceleration caused by road disturbances. The underlying motive for conducting a comparative study of various controllers' responses to speed bumps is because speed breakers are a common sight in India and also an indicator of disruption in road transport and safety.

\subsection{Contribution and paper structure}

In this paper, a control technique for linear time-invariant based semi-active suspension for a quarter car model are presented. The study focuses on bumps and humps, which are specified by the Indian government in section IRC 991988 of the Indian Road Congress and customary design approved by different manufacturers all over India. Each of the bumps and humps considered has a distinct shape

\footnotetext{
*e-mail: sarvesh.walavalkar@gmail.com

**e-mail: virajtan60@gmail.com

***e-mail: thakurrahul62690@gmail.com

****e-mail: pramodvepada99@gmail.com

†e-mail: supriya.bhuran@rait.ac.in
}

and height. According to safety standards, the Indian government has contemplated a certain highest arrival speed in which the heave motion would be minimum and considered to be safe for high-value cargo vehicles and ambulances. The article suggested does not take into account the speed component, but rather the response of the model to displacement caused by road disturbances. This study could be beneficial to automobile makers by offering a simple method of developing suspension systems for cars that deliver outstanding dampening response on government-approved speed bumps.

\section{Literature Review}

Many valuable contributions have recently been published in the literature for the design of semi-active suspension systems, with each publication focused on distinct characteristics such as invariable road disturbances, design framework and so on. Each paper has a distinct ideology to tackle the heave motion, and the intrusion created due to road profile. The passive system is considered in many research as of open-loop type and pairing it with any controller improves the transient response significantly. The advantage gained by this approach notably reduces peak overshoot, settling time, and other timedomain parameters[4]. Another approach to this problem is by implementing higher accuracy controller like Model predictive controller cascaded with the secondary controller, the output of semi-active system is much better than passive in invariable road disturbances by improving vertical chassis acceleration and peak chassis acceleration at bump points[6].

Logic-based controllers like fuzzy are proved to provide the best response in such random inference sys- 
tems. When fuzzy is optimized with the right optimization method, such as particle swarm optimization, the maximum peak overshoot and stabilization time are reduced [8]. Advanced research in this area has revealed that semiactive systems can be nonlinear and that by taking nonlinear factors into account, a fuzzy controller may be implemented on a nonlinear model to reduce heave, rolling, and pitch motion acting on the chassis[5].

A certain approach toward developing a cheap model of SAS is by using magnetorheological dampers(MR) much research is focused on developing a stable model of MR dampers and increasing its overall response. Considering a Bouc-Wen model of the MR damper can pivot the direction of the research to non linearities present in the model.Bouc-Wen model provides more insight on acceleration acting on various sections of the body. With a proper state space model a more accurate model can be designed and various parameters such as acceleration, velocities can be derived and reduced accordingly[12]. Complex controllers like fuzzy and sliding mode control can also be implemented on such systems. One of the approaches toward developing is to pair complex controllers with neural networking[1]. This strategy proves useful for annihilating a certain type of disturbance from the system. Variable universal fuzzy control, Fuzzy neural network and particle swarm optimization (VUFC-FNNO-PSO) are more effective to remove random road vibrations than passive. Particle swarm optimization provides backward integration to training and learning for the fuzzy neural network (FNN) which can improve the response further[13].

A new perspective towards this anomaly is by constructing a skyhook model. These models improve the researchers attitude towards ride comfort qualities. These methods improve the comfort quality by $24 \%$ than traditional systems without sacrificing the handling qualities[2][3]. This method is only useful for random noise generated by the road. Skyhook control algorithm maintains the degradation of handling performance within the range of $10 \%$ which improves the system significantly than the standard models[14]. Linear Parameter Varying (LPV ), and Frequency Estimation-Based (FEB) are some of the approaches to increase handling and road grip of the tires. Comfort improvements during the LPV approach are $36 \%$ greater in pitch reduction than the passive control strategies and $24 \%$ better in chassis control. Both controllers reduce the tire compression by $30 \%$ and reduce heave motion by $40 \%$ than any standard suspension system[15].

\section{Proposed Algorithms}

The study's approach is to use mechanical equations of the system to construct a proper and reliable model of the passive suspension system. Now, this passive model is converted into a linearized control system model to get the transfer function. Using this transfer function, the stability analysis of the model can be obtained. The transfer function provided the stability analysis and the value of damping coefficient (). The next step is to turn the open loop passive model into a closed loop model with a controller and a feedback element gain that can be cascaded. The reaction of the suspension system connected with the controller can be compared to the passive model response by picking a correct controller each time. If the controller's response is worse than the passive system's, it can be finetuned or paired with a different optimization strategy. The process continues until the desired response is achieved.

\section{Methodology}

A closed-loop system with feedback elements and external road disturbance can be simplified with the given block diagram in Fig. 1.

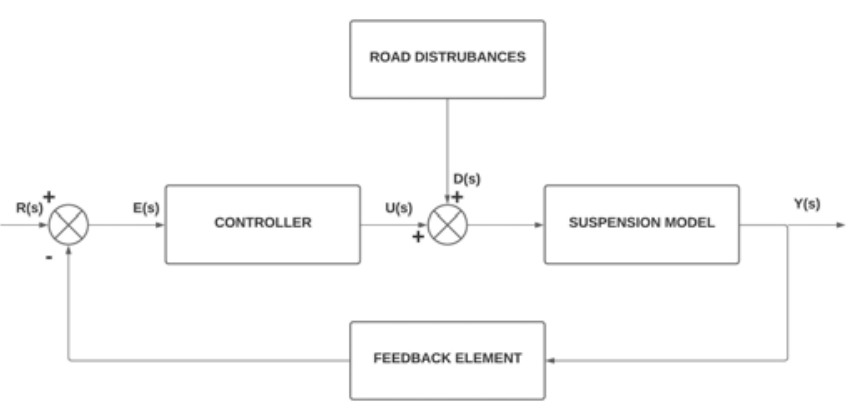

Figure 1. Block Diagram for closed loop system

Vertical motions and disturbances will be detected by sensors positioned on the vehicle's body. These values are sent to the controller, which will take corrective action to bring the system back into balance. The damping coefficient will be controlled directly by the controller by manipulating current values into system. Fig. 2. demonstrates the dynamic model for the quarter car system.

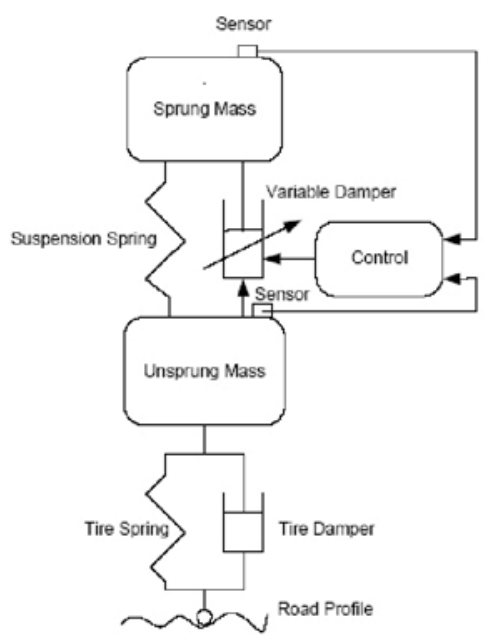

Figure 2. Dynamic model for Quarter car

\subsection{Passive Suspension System}

The characteristics of springs and dampers are fixed in the passive suspension system. The designer of the suspension determines these characteristics concerning for design goals and the desired application. Consider $M_{2}, M_{1}$, 
and $W$ to be wheel mass, vehicle mass, and tire contact point to the road respectively. An unrealistic assumption that the axles move in the same way is made considering the vertical movement of the car body and axles so that they are carried together and the rotational movement of the car body can be disregarded. Since the road is flat, we can consider the car body and the axle can move autonomously, $x_{1}$ and $x_{2}$ are reference variables which are position variables, the position of the car body (mass $M_{1}$ ), and of axle (mass $M_{2}$ ) under the impact of gravity of the normal road level. The tires are modeled as rigid with a high stiffness that is combined in parallel with a damper for energy dissipation through the rubber. A linear spring and a linear damper connecting the axle to the car body are used to model the main suspension systems, which include coil springs and shock absorbers. Let $x_{1}$ be the difference between the usual road level and the point of contact between the tire and the road. The profile of the road and the location of the car decide $x_{1}$. The tire spring force (in an upward direction) associated with the tire's deviation from the standard road level is low, while the corresponding frictional force is $b 2 W$. The state-space equations can be obtained by applying Newton's second and third law to Fig. 3.

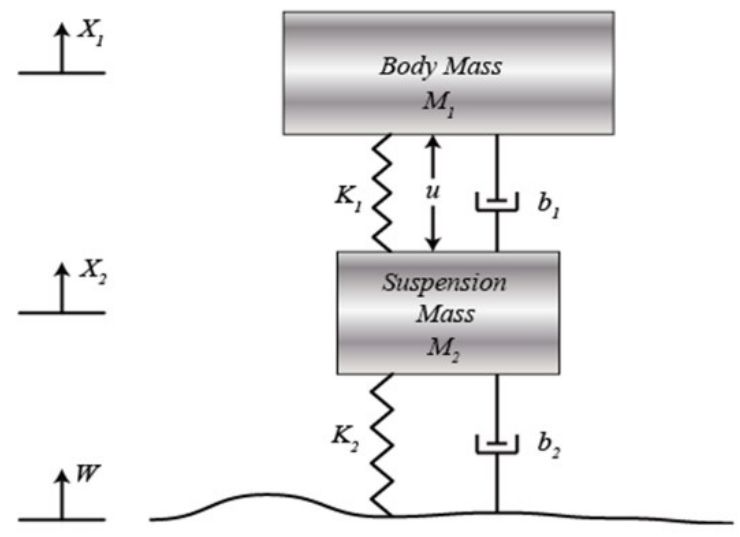

Figure 3. Semi - Active Suspension for Quarter car Model [4]

The equation of motion would be as follows if Newton's second law is applied to the two masses and Newton's third law is applied to the interaction between the two masses [4]:

$$
\begin{gathered}
M_{1} \ddot{x}_{1}+b_{1}\left(\dot{x}_{1}-\dot{x}_{2}\right)+k_{1}\left(x_{1}-x_{2}\right)=0 \\
M_{2} \ddot{x}_{2}+b_{1}\left(\dot{x}_{2}-\dot{x}_{1}\right)+k_{1}\left(x_{2}-x_{1}\right)+b_{2} \ddot{x}_{2}+k_{2} x_{2} \\
=b_{2} \dot{W}+k W \\
M_{1} S^{2} x_{1}(s)+b_{1} S\left(x_{1}(s)-x_{2}(s)\right)+k_{1}\left(x_{1}(s)-x_{2}(s)\right)=0
\end{gathered}
$$

$$
\begin{gathered}
2 S x^{2}(s)+k_{2} x^{2}(s)=b_{2} S W(s)+k_{2} W(s) \\
M_{1} \ddot{x}_{1}+\widetilde{b}_{1}\left(x_{1}-x_{2}\right)+k_{1}\left(x_{1}-x_{2}\right)=0 \\
M_{2} \ddot{x}_{2}+\widetilde{b}_{1}\left(\dot{x}_{2}-\dot{x}_{1}\right)+k_{1}\left(x_{2}-x_{1}\right)+b_{2} \dot{x}_{2}+k_{2} x_{2}=b_{2} \dot{W}+k_{2} W \\
M_{1} S^{2} x_{1}(s)+\widetilde{b}_{1} S\left(x_{1}(s)-x_{2}(s)\right)+k_{1}\left(x_{1}(s)-x_{2}(s)\right)=0 \\
M_{2} S^{2} x_{2}(s)+\widetilde{b}_{1} S\left(x_{2}(s)-x_{1}(s)\right)+k_{1}\left(x_{2}(s)-x_{1}(s)\right) \\
+b_{2} S x^{2}(s)+k_{2} x^{2}(s)=b_{2} S W(s)+k_{2} W(s)
\end{gathered}
$$

To obtain the transfer function, We have considered a linearized open-loop model while maintaining its linear invariant characteristics. The obtained Fourth order Transfer Function is

$$
\frac{422.1 s+8442}{s^{4}+44.93 s^{3}+555.5 s^{2}+1327 s+8442}
$$

The parameters considered are $M_{1}=241.5 \mathrm{~kg}$, $M_{2}=41.5 \mathrm{~kg}, K_{1}=6000 \mathrm{~N} / \mathrm{m}, K_{2}=14000 \mathrm{~N} / \mathrm{m}, b_{1}=300$ $\mathrm{N}-\mathrm{s} / \mathrm{m}, b_{2}=1500 \mathrm{~N}-\mathrm{s} / \mathrm{m}$

\subsection{Semi-Active Suspension with PID for Quarter Car Model}

Only the damping speeds, an embedded controller, and a series of sensors are handled by external power in the Semi-active suspension system. Based on a control strategy, the controller computes the amount of damping and automatically adjusts the damper to achieve the damping. The proportionality constants $\mathrm{Kp}, \mathrm{Ki}$, and $\mathrm{Kd}$ are determined by the method of trial and error.

When tuning controllers, deciding which controller to use is crucial. There are four basic control combinations: proportional $(\mathrm{P})$ only, proportional plus integral (PI), proportional plus derivative (PD), and proportional plus integral plus derivative (PID). For comparative analysis, we have used PI and PID controllers.

$K_{i}=35.2368, \quad K_{d}=23.6584, \quad$ and $K_{p}=50.3652$ are the integral, derivative, and proportionality constants, respectively[4].

\subsection{Semi - Active Suspension with IMC based PID (with and without filter) for Quarter Car Model}

The IMC-based PID method provides the same feedback output as IMC for stable processes with no time delay. The IMC-based PID procedure will not provide the same output as IMC for stable processes with a time delay since a time delay is introduced in Padé's deadtime approximation. Calculation of dead time from the $\operatorname{graph}(\theta p)$, and compared with standard equations and calculated gains and selected lambda from the given dead time observed with formulas. For IMC-based PID tuning, we have reduced the 4 th order transfer function to 2 nd order transfer function. To obtain 2nd-order transfer function we have observed dead $\operatorname{time}(\theta)$ from the graph. The selection of $\lambda$ and $\theta$ is essential[3].

Second order Transfer Function =

$$
\frac{1}{0.0557 s^{2}+0.0704 s+1}
$$


Standard equation $=$

$$
\frac{K p}{(\tau) s^{2}+2(\zeta)(\tau) s+1}
$$

$K_{i}=65.9198, K_{d}=45.6972, K_{p}=55.3254$, and $N=196.2526$ are the integral, derivative, proportionality constants, and Filter coefficient respectively.

\subsection{Semi-Active Suspension with Fuzzy controller for quarter car model}

A mathematical model of the system to be controlled is needed for classical control. However, since it is based on fuzzy logic which is shown in Table 1., it does not require a mathematical model. a structure based on rules, when applied to ill-defined and dynamic systems, FLC has an advantage over classical controllers[9].

The steps for developing a Fuzzy Logic-based suspension system are as follows:

- Fuzzification: the sharp inputs are converted into fuzzy outputs.

- Rule design: the truth values of the fuzzy output are computed,

- Computation: the requisite control function is computed, and

- Defuzzification: the fuzzy output is transformed into crisp values[1].

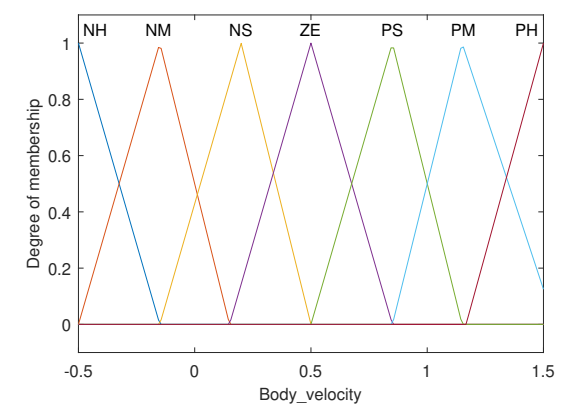

Figure 4. Membership Function of i/p variable "Body Velocity"

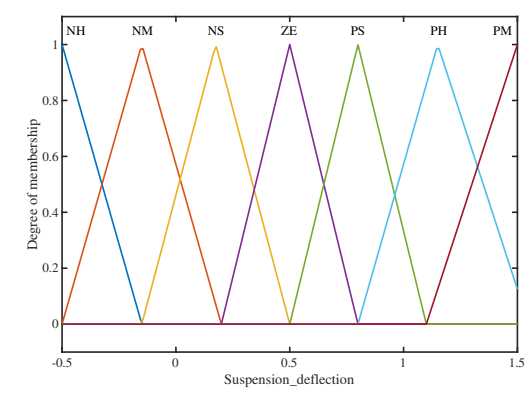

Figure 5. Membership Function of i/p variable "Suspension Deflection"

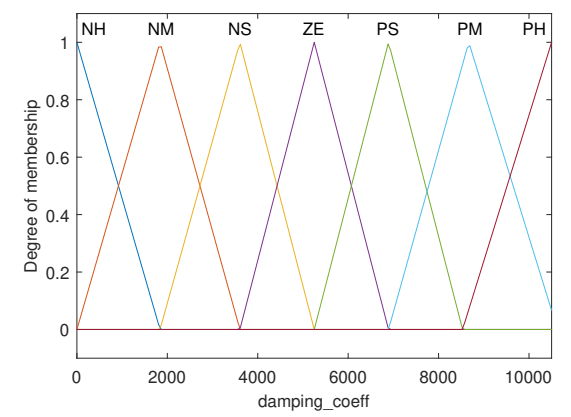

Figure 6. Membership Function of o/p variable "Damping Coefficient"

A membership function is a fuzzy set with pre defined range of 0 to 1 as shown in Fig 4,5 and 6, Fig. 7. explains the output surface of the proposed fuzzy system.

\begin{tabular}{|c|c|c|c|c|c|c|c|}
\hline & PL & PM & PL & ZE & NS & NM & NL \\
\hline NL & PM & PS & ZE & NS & NS & NS & NM \\
\hline NM & PM & PM & PS & NS & NM & NM & NM \\
\hline NS & PL & PL & PM & NS & NM & NM & NM \\
\hline ZE & PL & PL & PM & ZE & NM & NL & NL \\
\hline PS & PL & PM & PM & PS & NM & NL & NL \\
\hline PM & PM & PM & PM & PS & NS & NM & NM \\
\hline PL & PM & PS & PS & PS & ZE & NS & NM \\
\hline
\end{tabular}

Table 1. Fuzzy Rule Table [7]

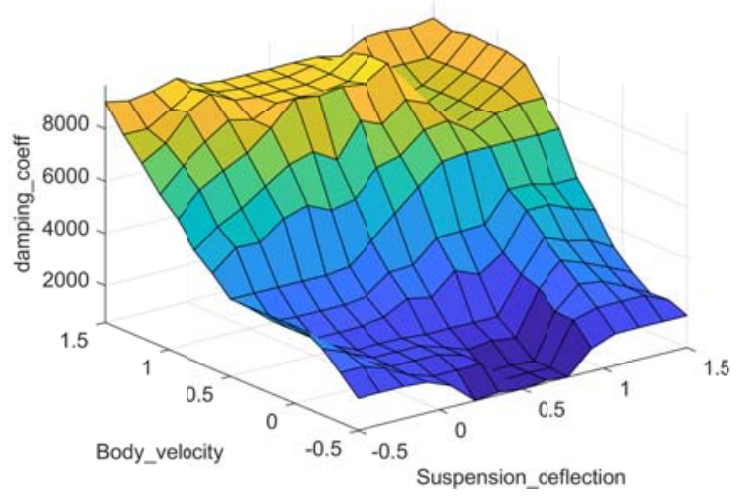

Figure 7. Surface view of Fuzzy i/p vs o/p functions

\subsection{Semi-Active Suspension with ANFIS for quarter car model}

The ANFIS controller combines the benefits of a fuzzy controller and a neural network. Fuzzy logic demonstrates computational effectiveness in integrating human expertise related to the control system being modelled, while neural networks are highly competitive in dealing with mathematical data. 
As a result, the ANFIS controller, when used in conjunction with a neural network and a fuzzy controller, has a higher capacity due to its ability to learn from mathematical results[10]. Based on the selected Membership fuctions shapes and input-output pairs, the structure and fuzzy if-then rules of FIS in the ANFIS controller are automatically constructed based on the neural network learning algorithm.[2] A membership function is a fuzzy set with pre-defined range of 0 to 1 as shown in Fig 8 and 9, Fig. 10. demonstrates the Surface view of ANFIS for $\mathrm{i} / \mathrm{p}$ vs o/p functions.

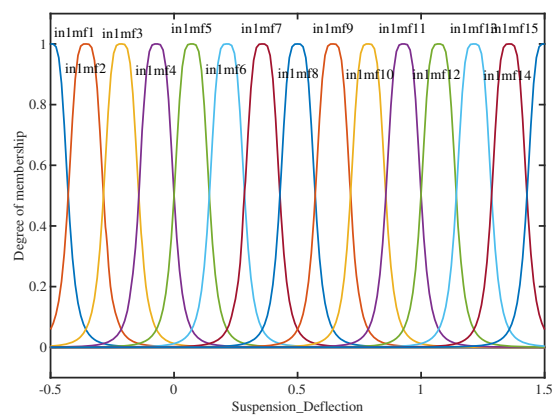

Figure 8. Membership Function of i/p variable "Suspension Deflection"

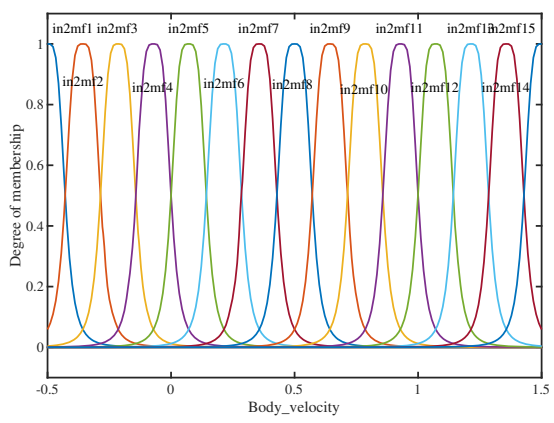

Figure 9. Membership Function of i/p variable "Body Velocity"

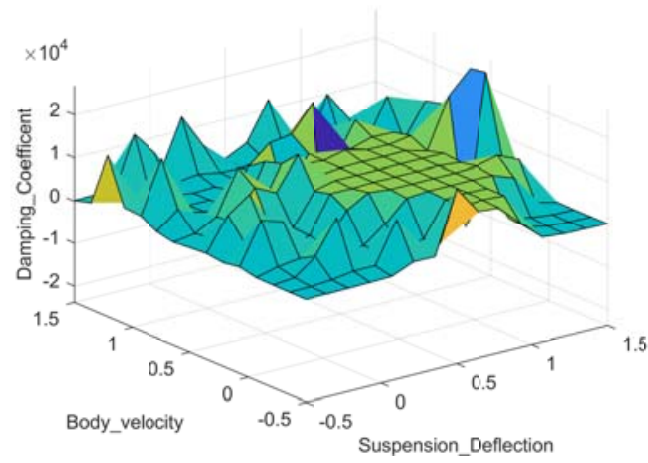

Figure 10. Surface view of ANFIS for $\mathrm{i} / \mathrm{p}$ vs o/p functions
A neuro-fuzzy system is a fuzzy system that determines its parameters (fuzzy sets and fuzzy rules) by processing data samples using a learning algorithm derived from neural network theory [11].

\section{Results \& Discussion}

Ride comfort is influenced by several elements while driving over a speed bump including vertical spring action, the oscillation that occurs during the settling process, the time required to settle after oscillatory behavior, and the maximum heave motion are typically associated with a smooth ride. The relatively low-frequency bounce and rebound motions of the suspension system are a role in the ride quality, which is typically linked with the vehicle's response to bumps. After a bump, a vehicle's undamped suspension (without variable shocks) will suffer a sequence of oscillations that will cycle according to the system's natural frequency.

Measuring and quantifying ride comfort not only assists development teams in satisfying essential standards and laws, but also provides the necessary insight to troubleshoot, comprehend, and improve the vehicle's noise and vibration comfort.

The handling characteristics of an automobile are concentrated on the characteristics of the dampers. They are the final link in the driver's chain of output directives, and they regulate the input of forces and disturbances from the road. The effect of the road on the vehicle, as well as the effectiveness of the output forces that control vehicle stability and cornering characteristics, are all influenced by damper properties.

The system of springs, mass, tire, and linkages that control how tires move and react to disturbances and control inputs manages the tire's basic characteristics. When a speed breaker is encountered, the oscillatory behavior that results might impair handling quality and could represent a threat if arrived at a specific speed.

The vehicle models created in MATLAB (Figures 4 and 5) are utilized to investigate the performance of the various controllers on disturbances in a realistic manner. These models simulate the behavior of a vehicle with continuous damping control. The results will be a form of linear displacement of the system after the disturbance has acted on the system such simulations have been carried out using the following benchmark speed breakers employed in industrial tests:

\subsection{Circular speed bump}

The performance of car suspension systems is put to the test with circular wave bumps. The test are simulated in such a way that pitch movements are successfully stimulated because the length of the hole is bigger than the distance between the front and rear axles (Gillespie, 1992). Furthermore, bounce performances may be assessed based on the amplitude characteristics of such road bumps.

The road disturbance/profile is taken according to Indian standards which are mentioned in Indian congress 
section IRC 99-198 as shown in Fig 11 and 13, Where ' $\mathrm{a}$ and b' denotes their bump amplitudes respectively, and their width are denoted as ' $c$ and d'. The frequencies of these bumps are $0.1 \mathrm{HZ}$ and $1 \mathrm{HZ}$. Fig 12 and 14 demonstrates the response of various controllers.

$$
W(x)=a 1[u(t-5)-u(t-10)] \sin (0.2 \pi t)
$$

$5 \leq \mathrm{t} \leq 10$

Where, $(\mathrm{a}=0.1 \mathrm{~m}$ (road bump height) and $\mathrm{c}=5 \mathrm{~m}$ (width) $)[4]$

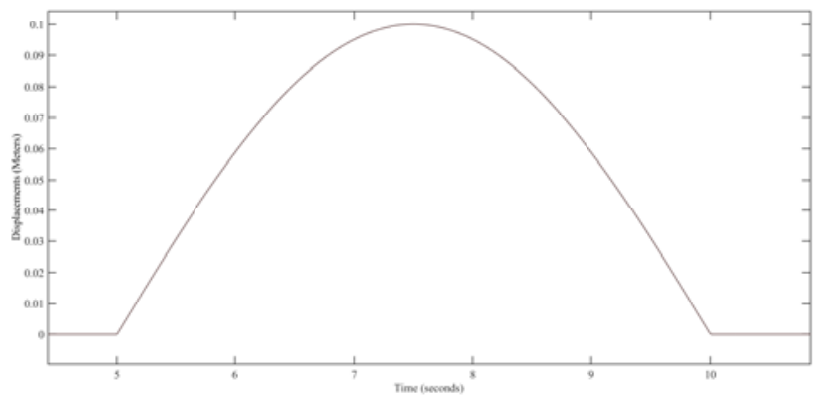

Figure 11. Road profile 1a

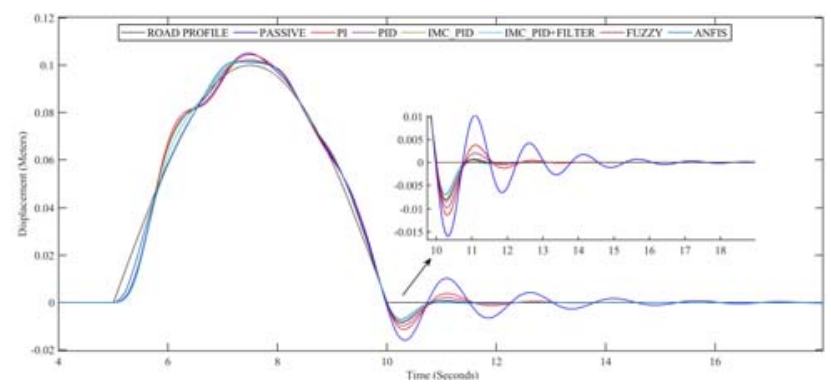

Figure 12. Response against Road profile 1a

$$
W(x)=a 1[u(t-30)-u(t-30.5)] \sin (2 \pi t)
$$

$30 \leq \mathrm{t} \leq 30.5$

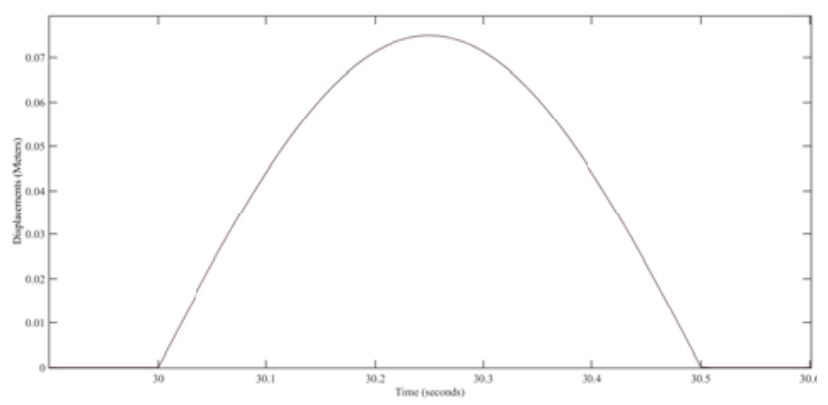

Figure 13. Road profile 1b

Where $b=0.75 \mathrm{~m}$ (road bump height), $d=0.5 \mathrm{~m}$ (width)

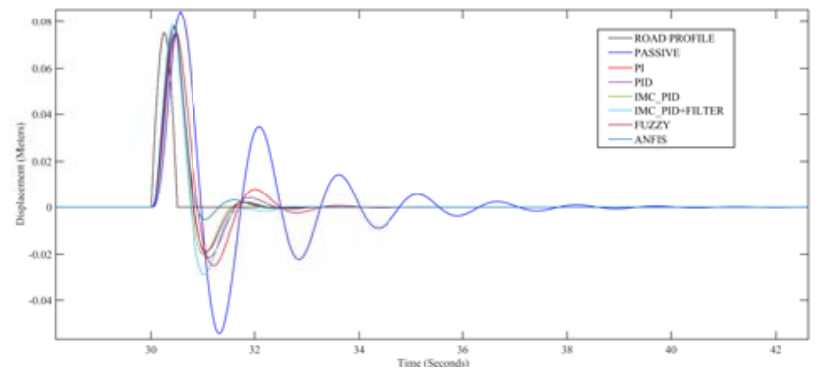

Figure 14. Response against Road profile $1 \mathrm{~b}$

\subsection{Sinusoidal speed bumps}

The sinusoidal curve is simulated with the help of the gaussian curve equation. For the desired signal curve, the values of sigma and $\mathrm{c}$ are considered accordingly. The height and width of the bump are denoted by $\mathrm{x}$ and $\mathrm{y}$ respectively. The road profile 2 (Fig 15) can be obtained from Equation 14 and Fig. 16. shows the response of various controllers against road profile 2 .

$$
W(x)=f(x ; \sigma, c)=e^{-\frac{(x-c)^{2}}{2 \sigma^{2}}}
$$

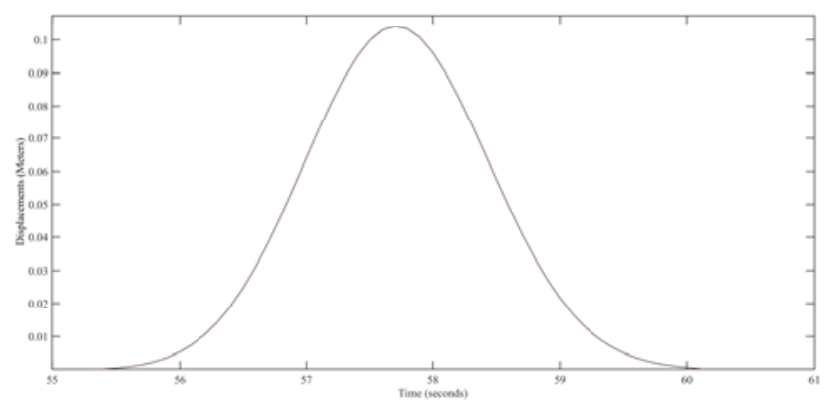

Figure 15. Road profile 2

Where, $(\mathrm{x}=0.1 \mathrm{~m}$ (road bump height), $\mathrm{y}=5 \mathrm{~m}$ (width)

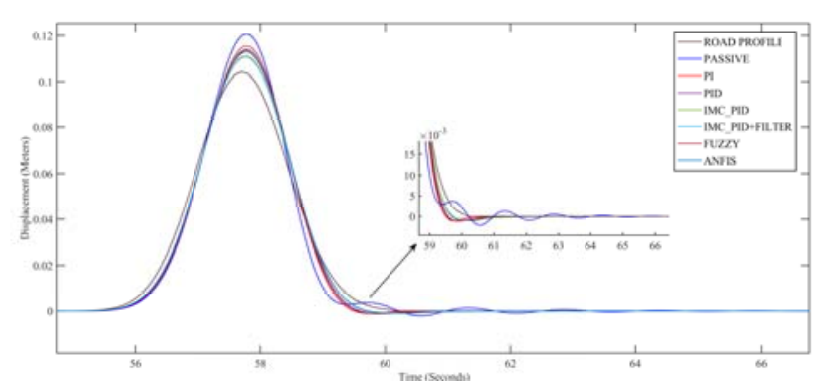

Figure 16. Response against Road profile 2

\subsection{Trapezoidal speed bumps}

The distinctive design of the trapezoidal speed bump assists drivers in reducing vehicle speed without compromising vehicle handling.The shape of the breaker is distinctive 
and can be visible from far distance providing users ample amount of time to reduce and control the speed of the vehicle. The features such as height and width are taken accordingly as vendors specified design.

The height and width of the bump is denoted by $\mathrm{p}$ and q respectively. The road profile 3 (fig 17) can be obtained using equation 15 and Fig. 18. demonstrates the response of various controllers against the road profile 3

$$
f(x)= \begin{cases}r(t-75) & 75 \leq \mathrm{t} \leq 76.2 \\ u(t-76.2) & 76.2 \leq \mathrm{t} \leq 78.8 \\ -r(t-78.8) & 78.8 \leq \mathrm{t} \leq 80 \\ 0 & \text { otherwise }\end{cases}
$$

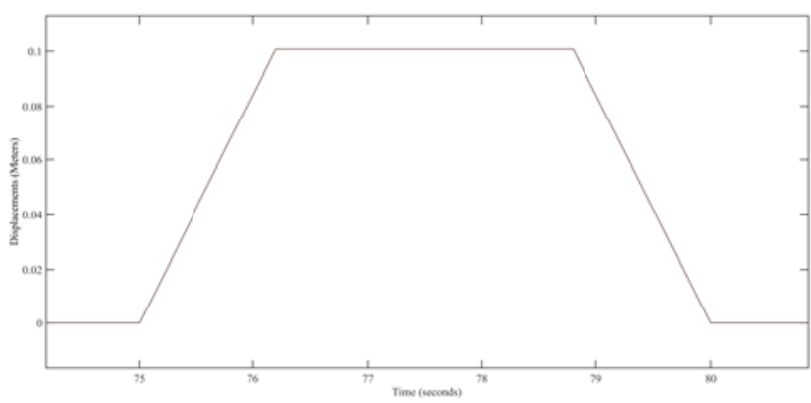

Figure 17. Road profile 3

Where, $(\mathrm{p}=0.1 \mathrm{~m}$ (road bump height), $\mathrm{q}=5 \mathrm{~m}$ (width))

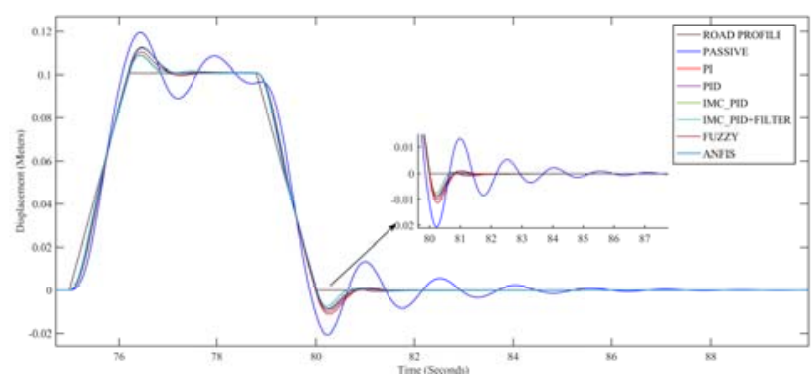

Figure 18. Response against Road profile 3

\subsection{Overall speed bump}

The design of the semi active suspension and its linear displacement caused due to bumps are focused individually to provide a more detailed view of the performance. The final perspective on the causes and effects is summed up by contemplating a road profile with these disturbances cascading to one another.

This outcome can be obtained in simulation by adding all of the responses together to obtain a final model of the road profile(Fig. 19).

$\mathrm{W}(\mathrm{x})=$ summation of all signal in equation

$$
\text { Adding equations }(12)+(13)+(14)+(15)
$$

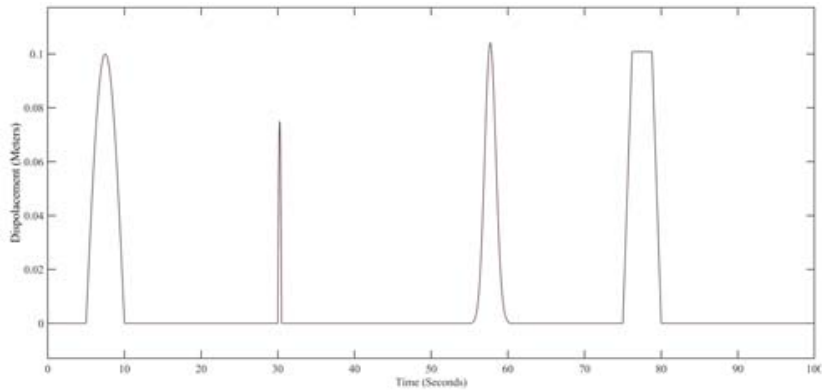

Figure 19. Road profile Overall

Fig. 20. is the response of various controllers against summation of road profiles

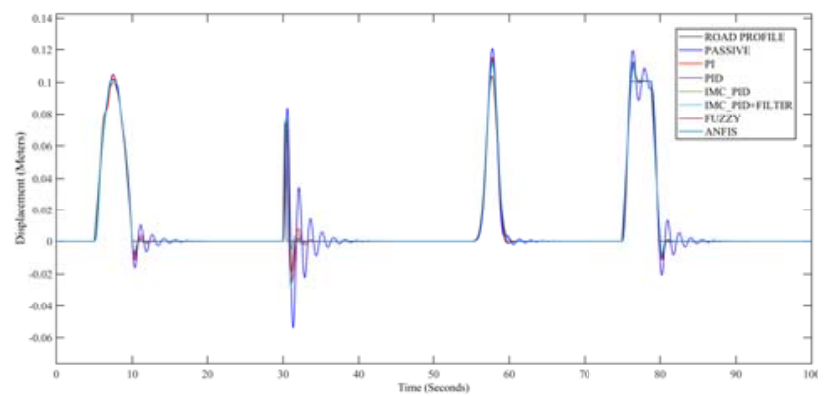

Figure 20. Response against Overall Road profile

The performance of the quarter car Semi-active suspension system is compared with the passive, PID controller, IMC based PID, IMC based PID with Filter, Fuzzy, and ANFIS controllers under various road profiles in this segment.

The immediate value of the controller output is defined as the control effort. It could be stated as an integral part of the power of a control signal. The controller effort gives you an idea of how different controllers compare the RMS value of the controller efforts of various controllers is shown in Table 2 throughout the simulation.

\begin{tabular}{ll}
\hline Controller & Controller Efforts \\
\hline Passive & $2.55 \mathrm{e}-5$ \\
PI & 3230.8172 \\
PID & 4810.9117 \\
IMC-PID & 7619.6825 \\
IMC-PID + Filter & 8910.2395 \\
FUZZY & 2445.9078 \\
ANFIS & 3007.8301 \\
\hline
\end{tabular}

Table 2. Controllers Efforts 


\begin{tabular}{llllll}
\hline Controller & ISE & ITAE & ITSE & IAE & Error parameter \\
\hline ANFIS & 0.0004325 & 2.384 & 0.01669 & 0.05286 & Suspension on deflection \\
& 0.08216 & 39.35 & 3.662 & 0.8668 & Body Velocity \\
FUZZY & 0.00041 & 2.50 & 0.01677 & 0.05512 & Suspension on deflection \\
& 0.08715 & 40.16 & 3.84 & 0.9005 & Body Velocity \\
IMC-PID & 787.7 & 4492 & $3.888 \mathrm{e}+04$ & 94.53 & Body Displacement \\
IMC-PID+Filter & 786.6 & 4506 & $3.881 \mathrm{e}+04$ & 94.93 & Body Displacement \\
PI & 803.2 & 4554 & $3.979 \mathrm{e}+04$ & 96.43 & Body Displacement \\
PID & 793.2 & 4515 & $3.925 \mathrm{e}+04$ & 95.2 & Body Displacement \\
\hline
\end{tabular}

Table 3. Performance Index

\begin{tabular}{llllllll}
\hline Parameter & PI & PID & IMC-PID & IMC-PID with filter & Passive & FUZZY & ANFIS \\
\hline RiseTime(ms) & 264.249 & 177.935 & 96.0865 & 95.1098 & 103.4889 & 48.9998 & 12.8251 \\
Settling Time(s) & 3.1248 & 2.256 & 2.084 & 1.840 & 8.564 & 1.642 & 1.4473 \\
Overshoot & 65.7 & 50.3148 & 60.6402 & 72.026 & 69.806 & 34.5382 & 45.6255 \\
\hline
\end{tabular}

Table 4. comparison table

\section{Conclusion}

This study compares the performance of damping control schemes such as PI, PID, IMC-PID, IMC-PID with Filter, FUZZY, and ANFIS for the quarter-car model. Simulations on the semi-active vehicle suspension system using MATLAB/Simulink is used to evaluate the performance of each semi-active control in terms of vertical displacement and suspension travel. Performance indices are also used to assess the performance of controllers. This research could be relevant in the future for designing real-time optimal nonlinear state feedback car suspension adjustment to improve ride comfort and stability.

\section{References}

[1] J. Wang and L. Sun, "Design of semi-active air suspension system based on backstepping sliding mode control," 2017 Chinese Automation Congress (CAC), 2017, pp. 4378-4382, doi: 10.1109/CAC.2017.8243550.

[2] E. Enders, P. Karle, G. Bonelli, D. Killian and G. Burkhard, "Modal Vertical Vehicle Dynamics Control for Semi-Active and Active Suspension Systems," 2020 Fifteenth International Conference on Ecological Vehicles and Renewable Energies (EVER), 2020, pp. 1-9, doi: 10.1109/EVER48776.2020.9242985.

[3] J. Wu, H. Zhou, Z. Liu and M. Gu, "Ride Comfort Optimization via Speed Planning and Preview SemiActive Suspension Control for Autonomous Vehicles on Uneven Roads," in IEEE Transactions on Vehicular Technology, vol. 69, no. 8, pp. 8343-8355, Aug. 2020, doi: 10.1109/TVT.2020.2996681.

[4] Gowda, Dr.Dankan and Chakrasali, Sadashiva. (2014). Comparative Analysis of Passive and SemiActive Suspension System for Quarter Car Model using PID Controller.
[5] Qazi, Abroon \& Khan, Dr. Afzal \& Khan, Muhammad \& Noor, Sahar. (2013). A Parametric Study on Performance of Semi-active Suspension System with Variable Damping Coefficient Limit. AASRI Procedia. 4. 154-159. 10.1016/j.aasri.2013.10.024.

[6] Karthik Murali Madhavan Rathai. Experimental Implementation of Model Predictive Control Scheme for Control of Semi-active Suspension System. ScienceDirect, 2019.

[7] Wen, Jinyu \& Liu, Ju \& Long, Yao \& Yao, Wei. (2016). Solution to short-term frequency response of wind farms by using energy storage systems. IET Renewable Power Generation. 10. 669-678. 10.1049/iet-rpg.2015.0164

[8] Abroon Jamal Qazi Afzal Khan, M. Tahir Khan, Sahar Noor. A Parametric Study on Performance of Semi-Active SuspensionSystem with Variable Damping Coefficient Limit ,2014.

[9] Kazima, Sosthene and Josee, Musabyimana and Hui, Xiong. (2018). Fuzzy Logic Controller for Semi Active Suspension Based on MagnetoRheological Damper. International Journal of $\mathrm{Au}-$ tomotive Engineering and Technologies. Volume 7. 10.18245/ijaet.438045.

[10] Hung, N.C., Nhung, N.T.B., Vu, L.T.Y., Khai, V.Q., Son, T.A., Thanh, T.Q., 2020. Apply a Fuzzy Algorithm to Control an Active Suspension in a Quarter Car by Matlab's Simulink. AMM 902, 23-32. https://doi.org/10.4028/www.scientific.net/amm.902.23

[11] Abellan-Nebot, Jose. (2010). A review of artificial intelligent approaches applied to part accuracy prediction. International Journal of Machining and Machinability of Materials. 8. 10.1504/IJMMM.2010.034486

[12] Yao, G. Z. et al. "MR damper and its application for semi-active control of vehicle suspension system." Mechatronics 12 (2002): 963-973. 
[13] Pang, Hui Liu, Fan Xu, Zeren. (2018). Variable universe fuzzy control for vehicle semi-active suspension system with MR damper combining fuzzy neural network and particle swarm optimization. Neurocomputing. 306. 10.1016/j.neucom.2018.04.055.

[14] Durieux, Olivier Abramov, Sergey Mannan, Samjid. (2009). Semi-active suspension system simulation using Simulink. International Journal of Engineering Systems Modelling and Simulation. 1. 101-114.
10.1504/IJESMS.2009.027573.

[15] Juan C. Tudon-Martınez, Rubén Morales-Menéndez, Ricardo Ramirez-Mendoza, Olivier Sename, Luc Dugard. A Comparison between a Model-free and Model-based Controller of an Automotive Semiactive Suspension System : Independent Wheelstations. IFAC Joint conference SSSC - 5th Symposium on System Structure and Control, Feb 2013, Grenoble, France. pp.869-874, 